Revista Latinoamericana de la Papa 23 (2): 66 - 73, 2019

ISSN: 1853-4961

http://ojs.papaslatinas.org/index.php/rev-alap/index

\title{
Treinta años de contribución de la Fundación PROINPA a la agricultura boliviana: Caso del cultivar de papa "Marcela"
}

\section{J. Gabriel ${ }^{1}$}

Recibido: 04/10/2019

Aceptado: 12/12/2019

Accesible en línea: Diciembre 2019

\section{Resumen}

Con el objetivo de hacer un reconocimiento a los investigadores bolivianos, que no siempre son mencionados, hemos escrito este documento sencillo pero significativo, en la cual se relata el caso del cultivar de papa "Marcela", que está siendo ampliamente utilizada en los valles y alturas de Tarija y Potosí principalmente, debido a su tolerancia a la sequía, resistencia al tizón tardío de la papa (Phytophthora infestans) y precocidad, con rendimientos que oscilan entre 15 a $20 \mathrm{t} \mathrm{ha}^{-1}$. Este cultivar fue asumido por el INIAF como estrategia alternativa para afrontar a disminuir los efectos del cambio climático en la producción de papa.

Palabras clave adicionales: Tolerancia, resistencia, precocidad, rendimiento, participativo.

Thirty years of the PROINPA Foundation's contribution to Bolivian agriculture: Case of "Marcela" potato crop

\section{Summary}

With the objective of recognizing Bolivian researchers, who are not always mentioned, we have written this simple but significant document, in which the case of potato cultivation "Marcela", which is being widely used in the valleys from Tarija and Potosí heights mainly, due to its tolerance to drought, resistance to late potato blight (Phytophthora infestans) and precocity, with yields ranging from 15 to $20 \mathrm{t} \mathrm{ha}^{-1}$. This cultivar was assumed by INIAF as an alternative strategy to deal with reducing the effects of climate change on potato production.

Additional keywords: Tolerance, resistance, precocity, performance, participatory.

\footnotetext{
* Autor para correspondencia. E-mail: julio.gabriel@unesum.edu.ec, j.gabriel@proinpa.org

1 Profesor-investigador, Carrera Agropecuaria, Facultad de Ciencias Naturales y de la Agricultura, Universidad Estatal del Sur de Manabí, Jipijapa, Ecuador.
} 


\section{Introducción}

En Bolivia la papa ocupa el primer lugar entre los tubérculos cultivados con una superficie aproximada de 140.000 ha e involucra aproximadamente a 200.000 agricultores en la producción que son el 30 a $40 \%$ del total de agricultores del país (Gabriel y Carrasco, 1998; Blajos et al. 2007; Zeballos et al., 2009). Es la principal fuente de alimentación e ingresos en Bolivia (Estrada et al., 1994; Fernández-Northcote et al., 1999), y se conoce que 114 municipios del país han priorizado la papa (Zeballos et al., 2009). La papa ocupa el segundo lugar a nivel nacional; la producción es aproximadamente de 750 mil toneladas al año, lo cual representa entre 300 a 600 millones de bolivianos (Blajos et al., 2007; Zeballos et al., 2009).

La producción y productividad son afectadas por diversos factores, entre los cuales se pueden mencionar las frecuentes heladas y las agudas sequías, la baja fertilidad de los suelos, la alta incidencia de plagas y enfermedades, la falta de cultivares con características de resistencia a factores abióticos y bióticos adversos y la escasa semilla de buena calidad. Dada esta situación, el gobierno boliviano, mediante el Ministerio de Asuntos Campesinos y Agropecuarios (MACA), consideró de alta prioridad reforzar los trabajos de investigación en el cultivo de la papa (Gandarillas y Devaux, 1990).

Para ello, se implementó en octubre de 1989 el Proyecto de Investigación de la Papa denominado PROINPA, que fue ejecutado por el Instituto Boliviano de Tecnología Agropecuaria (IBTA) y el Centro Internacional de la Papa (CIP) y financiado por el gobierno Suizo a través de la Cooperación Técnica Suiza (COTESU). El proyecto incluye tres actividades fundamentales:

Investigación y generación de tecnologías orientadas a identificar y resolver las limitantes de la producción de papa; (2) Incremento de la producción de tubérculos semillas de categoría prebásica y (3) Fortalecimiento de la capacidad institucional de los diversos programas de investigación (Gandarillas y Devaux, 1990).

El PROINPA realizó sus actividades de investigación a través de las Estaciones Experimentales del IBTA, en coordinación y colaboración con otras instituciones involucradas con el cultivo de la papa. La sede principal de sus actividades de investigación fue la Estación Experimental de Toralapa ubicada en el Departamento de Cochabamba a $3450 \mathrm{msnm}$ (Gandarillas y Devaux, 1990).

Para realizar sus planes de acción, el Proyecto fue estructurado en cinco unidades: Investigación, Investigación Regional, Información, Apoyo a la Investigación y Administración. La Unidad de Investigación fue constituida por siete departamentos, que ejecutaron proyectos de investigación para obtener alternativas tecnológicas a los problemas tanto de producción como de comercialización que enfrentan los agricultores. Las interacciones de los departamentos dentro de la Unidad de Investigación, favorecieron el desarrollo de una investigación interdisciplinaria que permitió interpretar mejor la problemática del sistema agrícola del productor de papa (Gandarillas y Devaux, 1990). Una de las unidades fue el departamento de Fitomejoramiento. En el mismo se trabajó en la obtención de 
cultivares adaptados al agro-ecosistema boliviano y resistentes o tolerantes tanto a los principales factores bióticos como abióticos, tales como el "rosario o falso nematodo del nudo" (Nacobbus aberrans), la verruga (Synchytrium endobioticum), el tizón tardío (Phytophthora infestans) y a las heladas (Gandarillas y Devaux, 1990).

Se trabajó en el restablecimiento del Banco Nacional de Papa en la Estación Experimental de Toralapa, como fuente de diversidad genética para el mejoramiento. Se definieron en el programa varias líneas de investigación como: rendimiento, resistencia al tizón tardío, a los nematodos (Nacobbus aberrans y Globodera sp.), a la verruga (Synchytrium endobioticum), a los virus (PVY y PLRV), a la polilla (Pthorimaea operculella), tolerancia a heladas, sequía y precocidad. Otras fuentes de resistencia provinieron de material genético del CIP y del ICA de Colombia. Las evaluaciones se realizaron en diferentes zonas paperas del país, para tizón (Cochabamba, Chuquisaca, Tarija y Valles Mesotérmicos de Santa Cruz), para nematodos (Cochabamba, Chuquisaca), para heladas (Cochabamba, Oruro, Potosí y La Paz) y para sequía (Cochabamba y Potosí).

En el periodo 1989 - 1997 el programa de mejoramiento genético de papa de PROINPA logró generar cultivares aptos para consumo en fresco y para la industria, que se están cultivando en varios sitios de Bolivia; entre las cuales se halla Robusta, Jaspe, Perla, India, Chaposa, Chota Nawi, Cordillera, Puka Huaycha, Aurora, Puyjuni Imilla, Palta Chola y Cholita Rosada, todas con resistencia al tizón y/o al nematodorosario (Carrasco et al. 1995; Gabriel et al. 2001; Gabriel 2007a; Gabriel et al. 2007b; Gabriel et al. 2007c), otras generadas fueron las tolerantes a la helada como Illimani, Tunari, Condori y Sajama (Gabriel et al. 2001), y finalmente algunos cultivares resistentes a verruga como Escalante y Pollerita.

En el año 1997, ocurrió un cambio fundamental, debido a que los institutos de investigación agrícola tenían que evolucionar de una estructura monolítica, por rubros, a un sistema de investigación más complejo, que involucra diversos sectores, entre ellos, al estado, a las universidades públicas y privadas, a las asociaciones de productores, a la empresa privada y a las ONGs. Esto implica la búsqueda de recursos financieros de diversas fuentes, nuevas alianzas de cooperación técnica y financiera, y cambios de organización para asegurar alta competitividad, respuesta a las demandas de tecnología y participación de usuarios en el financiamiento de tecnología (Gandarillas, 2019).

En este sentido, con una estructura institucional más moderna, competitiva y autónoma, se crea la Fundación para la Promoción e Investigación de Productos Andinos (PROINPA). La Fundación representa una alternativa para promover el desarrollo de los cultivos andinos en Bolivia, y fue recibido el respaldo del gobierno a través del Ministerio de Agricultura, Ganadería y Desarrollo Rural, enmarcándose en las estrategias de generación y transferencia de tecnología de ese ministerio. La fundación PROINPA, se constituye en un centro nacional de desarrollo tecnológico del cultivo de papa, quinua y otros cultivos andinos. Por la importancia estratégica que reviste el cultivo de la quinua para la seguridad alimentaria del altiplano $\mathrm{y}$ valles de Bolivia, y por el potencial de convertirse en un importante rubro de captación de divisas para el país, es que el Ministerio de Agricultura encomienda a 
la fundación continuar con los trabajos de generación y transferencia de tecnología y tomar responsabilidad de los convenios internacionales en este cultivo (Gandarillas, 2019).

En el año 1997 el IBTA fue cerrado, para evitar que se pierda toda la capacidad en desarrollo de tecnología que se había logrado, se constituyó la Fundación PROINPA (, que a pesar de ya no contar con un financiamiento estable, dio continuidad al programa de mejoramiento genético, manteniendo algunas líneas de investigación principales como son el rendimiento, la resistencia al tizón de la papa, al nematodo-rosario, al nematodoquiste, a los virus PVY y PLRV, y a la verruga con fondos gestionados de la cooperación internacional como la Unión Europea, FONTAGRO, PREDUZA, PRGA, BMZ, JANE, IFAD, CYTED, Consorcio Andino, INIA-España, etc.; para así continuar con la generación de nuevos cultivares (Gabriel, 2010).

Con los recursos disponibles por la captación de proyectos concursables que tienen una duración máxima de tres años, en el mejor de los casos, se continúa generando cultivares más productivos, resistentes a factores priorizados, precoces y con aptitud para consumo en fresco y la industria. Para lograr estas tecnologías se está utilizando material del banco de germoplasma de papas nativas y silvestres e incorporando clones avanzados del CIP y de otros países (Gabriel, 2010).

\section{Origen del cultivar Marcela}

El cultivar "Marcela" fue obtenido en el año 1990 por investigadores del entonces Programa de Investigación de la papa [Nelson Estrada (+), Julio Gabriel, Enrique Carrasco, Willman García y Osmar Mendoza], quienes en la Estación Experimental de Toralapa en
Cochabamba, Bolivia, hicieron cruzas entre un cultivar nativo llamado Sani Imilla (Solanum andigena), tetraploide tolerante a heladas de buena calidad culinaria y el cultivar Alpha (Solanum tuberosum), tetraploide de origen holandés precoz y de buena calidad para procesamiento. La nueva población fue denominada 90-184 (año - familia).

Los clones de esta familia fueron seleccionados inicialmente por tres años en Cochabamba en las zonas de Toralapa, Chullchunqani y Colomi para resistencia a tizón (Phytophthora infestans) y al nematodo del rosario (Nacobbus aberrans), logrando obtenerse varios clones, entre ellos el clon 90-184-1, llamado posteriormente como "Marcela". En el año 1994, fue enviado a Tarija (Estación Experimental de Iscayachi y la Huerta) como una familia de tubérculos, junto con otros 200 clones y seleccionado por ocho años con la participación activa de los Ingenieros Ricardo Casso, Hernán Cardozo y Jaime Herbas (Gabriel et al., 2011).

Fue presentada por el Instituto Nacional de Innovación Agropecuaria y Forestal (INIAF) un entidad pública del Estado Plurinacional de Bolivia, el 20 de abril del 2011 en la feria de la Madre Tierra en la Plaza Murillo de la ciudad de La Paz (Agronomía, 2019; y posteriormente el 31 de julio del 2014 en el programa con sello boliviano (INIAF, 2019).

\section{Un poco de historia}

En palabras textuales del Ing. Ricardo Casso menciona: "Nuestro cultivar llegó por el año 1994 en un lote de clones con el membrete de Familia de tubérculos para rendimiento y calidad", de la Estación Experimental Toralapa de Cochabamba, como clon 90-184-1, que es un código que los investigadores de PROINPA manejaban e indicaba el año 
de cruza, la familia a la que pertenecía y el clon o individuo seleccionado. Durante tres ciclos agrícolas en la Estación Experimental de Iscayachi, fue evaluado por su tolerancia a heladas, sequía, exceso de humedad $y$ ataque de plagas, juntamente con otros 200 clones. Solo diez clones fueron seleccionados $y$ tuvieron buenas características. Estos fueron llevados a parcelas de agricultores para realizar evaluaciones participativas. En la cosecha, las esposas de los agricultores, prepararon un hervido de papas con queso y unánimemente declararon al clon 90-184-1, como el que más se adecuaba a sus necesidades.

El nuevo cultivar nombrado "Marcela" se continuo cultivando los años siguientes, aunque no con mucho entusiasmo, posiblemente porque ni los agricultores ni los técnicos, vimos el potencial comercial del cultivar.

Los últimos cinco años, mediante un proyecto ejecutado por la Prefectura de departamento y la Fundación PROINPA, se hicieron parcelas para mejorar la producción de papa en la comunidad de La Huerta (provincia Arce). Como una de las propuestas tecnológicas para los agricultores, junto a otros cultivares $\mathrm{y}$ semilla de calidad, se llevó al cultivar Marcela. En la Huerta, el comportamiento del cultivar fue excelente y rápidamente cundió la noticia entre los agricultores y se dieron formas para conseguir algunos tubérculos como semilla.

La visión comercial que nos faltó con los agricultores de Iscayachi, surgió en $\mathrm{La}$ Huerta, nuestro cultivar tuvo un mercado interesante en Tarija y las otras ciudades del departamento, por ser un acompañamiento ideal para las carnes asadas y otros platos que son de alto consumo. La harinosidad de este cultivar y su alta calidad culinaria, la hacen bastante competitiva en el mercado. Esto hizo prever un futuro promisorio que tiene el mérito de haber sido seleccionado en nuestro medio y de acomodarse muy bien a las necesidades de los agricultores y también a las de los consumidores.

\section{Descripción del cultivar}

La planta del cultivar "Marcela" es herbácea con hábito de crecimiento erecto, los tallos son gruesos de color verde con poca pigmentación, alcanzando una longitud entre 0,90 a 1,20 metros. Sus hojas son compuestas y se distribuyen en espiral sobre el tallo. La forma de la hoja es disectada, con cinco pares de foliolos laterales y un par de interhojuelas sobre los peciólulos (Galarza 2003, INIAF 2019).

Tiene floración profusa, las flores son lila $\mathrm{y}$ con color secundario rojo-rosado $\mathrm{y}$ acumen blanco en el envés y forman bayas. Los estolones ligeramente cortos y pegados al tallo. Los tubérculos son redondos comprimidos, con ojos profundos y en la parte del ojo apical es semi-profundo (Gabriel et al., 2011).

La piel del tubérculo es de color rosado con color secundario blanco - crema; la carne es de color blanco. El brote es cónico y de color rosado.

Tiene un amplio rango de adaptación (1.600 a $3.300 \mathrm{msnm})$. Su rendimiento promedio experimental fue de 30 a $50 \mathrm{t}$ $\mathrm{ha}^{-1}$.

Es un cultivar harinoso, de rápido cocimiento y de presentación atractiva. Las primeras experiencias demostraron no ser apta para elaboración de papa frita.

\section{Difusión del cultivar de papa Marcela}

Hace 14 años se organizó el Instituto Nacional de Innovación Agrícola Forestal (INIAF), entidad dependiente del Ministerio de Desarrollo Rural y Tierras 
(MDRyT) que actúa bajo supervisión del Viceministerio de Desarrollo Rural y Agropecuario (VDRA).

El INIAF presentó un nuevo cultivar de papa denominado "Marcela", que como se conoce fue una cruza realizada en el año 1990 por el entonces Programa de Investigación de la Papa del ex - IBTA, y que fue seleccionado exitosamente en el departamento de Tarija y tiene la capacidad de producir dos veces al año (Gabriel et al., 2011).

Este cultivar de papa tiene similares características a cualquier otra, pero es de rápida cocción, de buen sabor y tamaño normal. Todas estas cualidades hacen muy atractivo su cultivo y consumo, afirmó el entonces director nacional de Investigación del INIAF, Celso Ayala (Agronomía, 2019). Mencionó que otra de sus cualidades es que tiene un potencial de rendimiento de hasta 40 t/ha (en condiciones adecuadas), un periodo vegetativo corto de 150 días (por lo que se puede producir y cosechar dos veces al año) y amplio rango de adaptación a la altitud, desde los 600 a 3.300 msnm.

Ayala explicó que el cultivar "Marcela" reacciona de forma más consistente a factores adversos, es resistente al tizón tardío de la papa (Phytophthora infestans) y presenta mayor tolerancia a las heladas, factores que comúnmente ocasionan importantes pérdidas en las parcelas de cultivo y afectan la producción y la economía de los agricultores. Este nuevo cultivar fue registrado en el año 2015 (INIAF, 2015)

\section{Impacto potencial del cultivar de papa "Marcela"}

Ayala aclaró que en Tarija cerca del 50 por ciento de la población agraria se dedica a la producción de este nuevo cultivar de papa sobre una extensión de
10.000 mil hectáreas, con un volumen promedio de 66000 toneladas y un rendimiento promedio de 6,6 toneladas por hectárea.

Para continuar en la mejora de la semilla de la papa denominada Marcela, Ayala anticipó que se tiene previsto efectuar ensayos, pruebas y experimentos en los valles templados y húmedos del altiplano y en el departamento de Santa Cruz, considerando la época de siembra y cosecha en ambos casos.

David Coronel del Instituto Nacional de Innovación Agropecuaria y Forestal (INIAF), dependiente del Ministerio de Desarrollo Rural y Tierras de Bolivia, informó de que este tubérculo se llega a cosechar hasta 20 toneladas por hectárea en comunidades del centro potosino (Agraria.pe., 2019).

\section{Reflexiones finales}

Hemos escrito este sencillo documento, como un reconocimiento a los investigadores involucrados a lo largo de estos 30 años de vida institucional. Estos investigadores que en su tiempo no siempre fueron reconocidos por la sociedad, las instituciones y las generaciones futuras. Creemos que es un justo homenaje y agradecimiento por haber contribuido con una tecnología que está siendo utilizado por las nuevas generaciones para alimentar y mejorar la economía de cientos y miles de agricultores. Esto nos hace ver que en la vida, las personas pasamos, pero las instituciones siguen; sembramos pero otros cosechan. Al final vemos que al menos tenemos la satisfacción de haber cumplido con nuestro pueblo y su inversión no fue vana, a pesar de los muchos años que pasaron. 


\section{Conflictos de intereses}

Los autores declaramos que este artículo no tiene conflicto alguno de interés.

\section{Referencias citadas}

Agraria.pe. (2019). Proponen cultivo de papa marcela contra cambio climático. Disponible en

https://agraria.pe/noticias/proponencultivo-de-papa-marcela-contra-11099.

Agronomía (2019). Marcela, una variedad de papa llenará los platos. Disponible en http://agroingeniero.blogspot.com/2011/0 4/marcela-una-variedad-de-papallenara.html.

Blajos, J.; Amaya, N.; Gandarillas, A. (2007). ¿Cuán importante es la papa para los bolivianos?. Revista de Agricultura 40 (59):7-9

Carrasco, E.; Estrada, N.; Gabriel, J.; Alfaro, G.; Larondelle, Y.; García, W.; Quiroga, O. (1997). Seis cultivares potenciales de papa con resistencia al tizón (Phytophthora infestans) en Bolivia. Revista Latinoamericana de la Papa 9 (10): 106-122.

Carrasco, E.; Estrada, N.; Gabriel, J.; García, W.; Mendoza, O.; Quiroga, O. (1995). Seis nuevas variedades de papa resistentes al tizón (Phytophthora infestans). Tecno-IBTA 5:1-8.

Estrada, N.; Fernández-Northcote, E.; Carrasco, E.; Navia, O. (1994). Mejoramiento genético para resistencia a enfermedades y plagas de la papa en Bolivia.. L.H.M. Broers (Ed.) en: Resistencia duradera en cultivos alto andinos, Memorias del 1er taller sobre Resistencia Duradera en Cultivos Alto Andinos. 30 de mayo-3 de junio de 1994. Quito, Ecuador p. 86-89

Fernández-Northcote, E.; Navia, O.; Gandarillas A. (1999). Bases de las estrategias de control químico del tizón tardío de la papa desarrolladas por PROINPA en Bolivia. Revista Latinoamericana de la papa 11:1-25.

Gabriel, J. (2010) Documento marco: Estrategias y perspectivas del mejoramiento genético de papa (Solanum tuberosum L.) en Bolivia. Fundación PROINPA, Cochabamba, Bolivia. 60 p.

Gabriel, J.; Carrasco, E. (1998) Evaluación preliminar de la resistencia durable al tizón Phytophthora infestans en cultivos nativos de papa del Banco de Germoplasma Bolivian. In Daniel Danial y Oswaldo Chicaiza (Ed.), Segundo Taller de PREDUZA en Resistencia Duradera en cultivos Altos en la Zona Andina. 22 - 24 septiembre de 1998. Cochabamba, Bolivia. p 153-158

Gabriel, J.; Carrasco, E.; García, W.; Equize, H.; Thiele, G.; Torrez, R.; Ortuño, N,; Navia, O.; Franco, J.; Estrada, N. (2001). Experiencias y logros sobre mejoramiento convencional y selección participativa de cultivares de papa en Bolivia. Revista Latinoamericana de la Papa 1: 169-192.

Gabriel' J.; Coca, A.; Plata, G.; Parlevliet, J.E. (2007a). Characterization of the resistance to Phytophthora infestans in local potato cultivars in Bolivia. Euphytica 153: 321-328.

Gabriel, J.; Vallejos, J.; Coca, C.; López, L.; Escobar, F.; Villarroel, E.; Villarroel, J. (2007b). Nuevas variedades de papa obtenidas por fitomejoramiento participativo. Revista de Agricultura 41 (59): $41-44$.

Gabriel, J.; Forqueda, F.; Plata, G.; Fernández-Northcote, E.N. (2007c). Caracterización de genotipos de papa de Europa y Latinoamérica por resistencia a tizón y propiedades culinarias. Revista Latinoamericana de la Papa 14 (1): 10-23. 
Gabriel, J.; Pereira, R.; Gandarillas, A. (2011). Catálogo de nuevas variedades de papa en Bolivia. Fundación PROINPA, Cochabamba, Bolivia. 55 p.

Galarza-Acosta, D. (2003). Caracterización morfología y agronómica de los cultivares de papa en las zonas productora del departamento de Tarija. Tesis presentada para obtener el grado Licenciatura en Ingeniería Agronómica. Universidad Juan Misael Saracho, Tarija, Bolivia. 74 p.

Gandarillas, A. (2019) Nueva estructura de generación y transferencia de tecnología en quinua en Bolivia. http://www.fao.org/tempref/gi/reserved/ft p_faorlc/old/prior/segalim/prodalim/prod veg/cdrom/contenido/libro14/cap4.2.htm
Gandarillas, A.; Devaux, A. (1990). Organización y funciones del nuevo Proyecto de Investigación de la Papa (PROINPA) de Bolivia. Revista Latinoamericana de la Papa 3:91-95.

INIAF (2015). Registro nacional de variedades 2015. Dirección Nacional de Semillas, INIAF, La Paz, Bolivia. https://portal.iniaf.gob.bo/publicacionessemillas/registro-nacional-de-variedadesy-de-variedades-protegidas-2015/

INIAF (2019). Variedad de papa "Marcela". Disponible en https://portal.iniaf.gob.bo/variedad-depapa-marcela/.

Zeballos, H.; Balderrama, F.; Condori, B.; Blajos, J. (2009) Economía de la papa en Bolivia (1998-2007). Fundación PROINPA, Cochabamba, Bolivia. 129 p. 